\title{
Methods and devices for protein crystal growth in space
}

\author{
Hiroaki Tanaka ${ }^{a}$, Sachiko Takahashia , Bin Yana, Misako Koga ${ }^{a}$, Yoshinobu Hashizume ${ }^{a}$, \\ Masayuki Kamob, Naoki Furubayashib and Koji Inakab
}

${ }^{a}$ Confocal Science Inc., 2-12-2 Iwamoto-cho, Chiytoda-ku, Tokyo 101-0023 JAPAN

bMaruwa Foods and Biosciences Inc., 170-1 Tsutsui-cho, Yamatokoriyama, Nara 639-1123 JAPAN

Email Contact: tanakah@confsci.co.jp

JAXA/NASDA has been conducting protein crystal growth (PCG) experiment in microgravity since 1992 (FMPT (STS-47)). And in 2003, JAXA has started to provide regular opportunities of crystallization experiment in microgravity using Russian vehicles such as the Soyuz and Progress; JAXA PCG [1]. Since then, we have technically supported users of JAXA PCG for nearly 20 years and incrementally improved our technologies to obtain high-quality protein crystals in microgravity [2]. We have tried more than one thousand conditions totally. Through the experiences, to obtain positive results from crystallization experiment in microgravity, we realize that crystallization technologies specialized for microgravity environment should be considered. We found out that if the protein sample is highly purified and the crystallization conditions are well-optimized, the maximum resolution and/or the morphology of the crystals can be improved with high reproducibility, which are the significant advantages of the PCG in microgravity $[3,4]$.

In JAXA PCG experiments, we developed a crystallization device, JCB-SGT, which is the primary crystallization container. It is very simple, versatile and has a wide range of applications. Various crystallization methods have been applied in JCB-SGT: counter-diffusion method, dialysis method vapor-diffusion method, and osmosis-tube method, using many varieties of capillary diameter [5]. Especially for growing large crystals, we developed LC (Large Capillary) and LCDM (Large Capillary with Dialysis Membrane) since there are a lot of requests from users to grow large crystals.

In this presentation, the outline of JCB-SGT, and its capillary configuration are introduced. And important insights and lessons learned from our experiences in optimizing crystallization conditions especially for microgravity experiments are explained. Our technology for successful crystallization in microgravity must be useful even for the crystallization experiments in the laboratory on the ground.

\section{$\underline{\text { References }}$}

[1] Yoshizaki I., et al., (2019) Int. J. Microgravity Sci. Appl., 36(1), 360101.

[2] Inaka K., et al., (2017) Int. J. Microgravity Sci. Appl., 34(1), 340104.

[3] Takahashi S., et al., (2010) Acta Cryst. F66, 846

[4] Inaka K., et al., (2011) Cryst. Growth Des. 11, 2107

[5] Takahashi S., et al., (2019) Int. J. Microgravity Sci. Appl., 36(1), 360107 